\title{
Symmetries, Lagrangian Formalism and Integration of Second Order Ordinary Difference Equations
}

Vladimir DORODNITSYN* ${ }^{*}$ Roman KOZLOV ${ }^{\dagger}$ and Pavel WINTERNITZ $\ddagger$

* Keldysh Institute of Applied Mathematics of Russian Academy of Science, Miusskaya Pl. 4, Moscow, 125047, Russia;

E-mail:dorod@spp.Keldysh.ru

$\dagger$ Department of Informatics, University of Oslo, 0371, Oslo, Norway;

E-mail:kozlov@ifi.uio.no

$\ddagger$ Centre de Recherches Mathématiques, Université de Montréal, Montréal, QC, H3C

3J7, Canada;

E-mail: wintern@crm.umontreal.ca

This paper is part of the Proceedings of SIDE V;

Giens, June 21-26, 2002

\begin{abstract}
An integration technique for difference schemes possessing Lie point symmetries is proposed. The method consists of determining an invariant Lagrangian and using a discrete version of Noether's theorem to obtain first integrals. This lowers the order of the invariant difference scheme.
\end{abstract}

\section{Introduction}

A recent article was devoted to a symmetry classification of second order difference equations [1]. This was modeled on a paper by Sophus Lie, in which he provided a symmetry classification of second order differential equations (ODEs) [2]. As a matter of fact, the classification of difference schemes goes over into Lie's classification of ODEs in the continuous limit [1].

S. Lie showed that a second order ODE can be invariant under a group $G_{r}$ of dimension $r=0,1,2,3$, or 8 . For $r \geq 2$ the equation can be integrated in quadratures. This can be done by transforming the equation to one of the "canonical" forms, integrated by Lie himself [2]. Virtually all standard methods of integrating second order ODEs 
analytically can be interpreted in this manner (though this is not mentioned in most elementary textbooks).

The situation with difference equations is much less developed. This is not surprising, since applications of Lie group theory to difference equations are much more recent $[3, \ldots, 27]$. Several different approaches are being pursued. One possibility is to consider the difference equations on a fixed lattice $[3, \ldots, 13]$ and consider only transformations that do not act on the lattice. In order to obtain physically interesting symmetries in this approach, it is necessary to go beyond point symmetries and to let the transformations act on more than one point of the lattice. Lie algebra contractions occur in the continuous limit and some "generalized" symmetries may "contract" to point ones [10].

The second possibility is to consider group transformations acting both on the difference equations and on the lattice $[1,17, \ldots, 27]$. For ordinary difference equations this is achieved by considering a difference scheme, consisting of two equations, one representing the actual difference equation, the other the lattice.

This is the approach that we will follow in the present article. More specifically, we will consider the same three-point scheme as in our article [1]. The continuous limit of the scheme, if it exists, will be a second order ODE.

Thus, we consider two variables, $x$ and $y$, with $x$ the independent one and $y$ dependent. The variable $x$ runs through an infinite set of values $\left\{x=x_{k}, k \in \mathbb{Z}\right\}$ that are not necessarily equally spaced and are not prescribed a priori. Instead, we give two relations between any three neighboring points

$$
\begin{aligned}
& F\left(x, x_{-}, x_{+}, y, y_{-}, y_{+}\right)=0 \\
& \Omega\left(x, x_{-}, x_{+}, y, y_{-}, y_{+}\right)=0
\end{aligned}
$$

and also specify some initial conditions like $x_{0}, x_{1}, y_{0}=y\left(x_{0}\right), y_{1}=y\left(x_{1}\right)$. In the continuous limit Eq. (1.1) goes into an ODE, (1.2) into an identity (like $0=0$ ), if the continuous limit exists.

The group transformations considered in this approach are of the same type as for ODEs. They are generated by a Lie algebra of vector fields of the form

$$
X=\xi(x, y) \frac{\partial}{\partial x}+\eta(x, y) \frac{\partial}{\partial y} .
$$

The corresponding transformations are purely point ones, since the coefficients $\xi$ and $\eta$ depend only on $(x, y)$, not on the shifted points $\left(x_{+}, y_{+}\right)$or $\left(x_{-}, y_{-}\right)$.

The purpose of this article is to provide a Lagrange formalism and difference analog of Noether's theorem for second order difference schemes of the form (1.1) and (1.2), admitting Lie point symmetry groups. The Lagrangians will be used to obtain first integrals and to obtain exact analytic solutions of the difference schemes. 


\section{Definitions and notations}

We study the difference system (1.1) and (1.2). In general, we assume that these equations can be solved to express $x_{+}$and $y_{+}$explicitly in terms of $\left(x, y, x_{-}, y_{-}\right)$and also vice versa, i.e. $\left(x_{-}, y_{-}\right)$in terms of the other quantities. We also make use of the following quantities

$$
\begin{aligned}
& h_{+}=x_{+}-x, \quad h_{-}=x-x_{-}, \quad y_{x}=\frac{y_{+}-y}{h_{+}}, \quad y_{\bar{x}}=\frac{y-y_{-}}{h_{-}}, \\
& y_{x \bar{x}}=\frac{2}{h_{+}+h_{-}}\left(y_{x}-y_{\bar{x}}\right),
\end{aligned}
$$

i.e. the up and down spacings in $x$, the right and left discrete first derivatives and the discrete second derivative, respectively. It is also convenient to use the following total shift and discrete differentiation operators:

$$
\underset{ \pm h}{S} f(x)=f\left(x_{ \pm}\right), \quad \underset{ \pm h}{D}=\frac{\stackrel{S}{ \pm}-1}{h_{+}}
$$

Continuous first and second derivatives are denoted $y^{\prime}$ and $y^{\prime \prime}$, respectively.

When acting on differential equations, the vector fields (1.3) must be prolonged to act on derivatives. For difference schemes, the prolongation of a vector field acts on variables at other points of the lattice. It is obtained by shifting the coefficients to the corresponding points. For three point schemes we have

$$
\text { pr } X=X+\xi\left(x_{-}, y_{-}\right) \frac{\partial}{\partial x_{-}}+\xi\left(x_{+}, y_{+}\right) \frac{\partial}{\partial x_{+}}+\eta\left(x_{-}, y_{-}\right) \frac{\partial}{\partial y_{-}}+\eta\left(x_{+}, y_{+}\right) \frac{\partial}{\partial y_{+}} \text {. }
$$

\section{$3 \quad$ Lagrangian formulation for second order ODEs}

It has been known since E. Noether's fundamental work that conservation laws for differential equations are connected with their symmetry properties $[28, \ldots, 31]$. For convenience we present here some well-known results adapted to the case of second order ODEs.

Let us consider the functional

$$
\mathbb{L}(y)=\int_{I} L\left(x, y, y^{\prime}\right) d x, \quad I \subset \mathbb{R}^{1},
$$

where $L\left(x, y, y^{\prime}\right)$ is called a first order Lagrangian. The functional (3.1) achieves its extremal values when $y(x)$ satisfies the Euler-Lagrange equation

$$
\frac{\delta L}{\delta y}=\frac{\partial L}{\partial y}-D\left(\frac{\partial L}{\partial y^{\prime}}\right)=0, \quad D=\frac{\partial}{\partial x}+y^{\prime} \frac{\partial}{\partial y}+y^{\prime \prime} \frac{\partial}{\partial y^{\prime}}+\cdots
$$


where $D$ is the total derivative operator. The equation (3.2) is an ODE that can be rewritten as

$$
y^{\prime \prime}=f\left(x, y, y^{\prime}\right)
$$

Let us consider a Lie point transformation $G$ generated by the vector field (1.3). The group $G$ is a "variational symmetry" of the functional $\mathcal{L}$ if and only if the Lagrangian satisfies

$$
\operatorname{pr} X(L)+L D(\xi)=0,
$$

where $\operatorname{pr} X$ is the first prolongation of the vector field $X$ to $y^{\prime}$. We will actually need a weaker invariance condition than given by Eq. (3.4). The vector field $X$ is an "infinitesimal divergence symmetry" of the functional $\mathbb{L}(y)$ if there exists a function $V(x, y)$ such that [29]

$$
\operatorname{pr} X(L)+L D(\xi)=D(V), \quad V=V(x, y) .
$$

The two important statements for us are:

1. If $X$ is an infinitesimal divergence symmetry of the Lagrangian $L$, it generates a symmetry group of the corresponding Euler-Lagrange equation. The symmetry group of Eq. (3.2) can of course be larger than the one generated by symmetries of the Lagrangian.

2. Noether's theorem $[28, \ldots, 31]$ can be based on the following Noether-type identity [31], which holds for any vector field and any function $L$ :

$$
\operatorname{pr} X(L)+L D(\xi)=\left(\eta-\xi y^{\prime}\right) \frac{\delta L}{\delta y}+D\left(\xi L+\left(\eta-\xi y^{\prime}\right) \frac{\partial L}{\partial y^{\prime}}\right) .
$$

It follows that if $X$ is a divergence symmetry of $L$, i.e. (3.4) or (3.5) is satisfied, then there exists a first integral

$$
J=\xi L+\left(\eta-\xi y^{\prime}\right) \frac{\partial L}{\partial y^{\prime}}-V=\text { const }
$$

of the corresponding Euler-Lagrange equation.

The above considerations tell us how to obtain invariant ODEs and conservation laws from divergence invariant Lagrangians. They do not tell us how to obtain invariant Lagrangians for invariant equations. This amounts to "variational integration", as opposed to variational differentiation.

A procedure that we shall use below to find invariant Lagrangians for differential equations can be summed up as follows.

1. Start from a given ODE $y^{\prime \prime}=f\left(x, y, y^{\prime}\right)$ and its symmetry algebra with basis

$$
X_{\alpha}=\xi_{\alpha}(x, y) \frac{\partial}{\partial x}+\eta_{\alpha}(x, y) \frac{\partial}{\partial y}, \quad \alpha=1, \ldots, k .
$$


Find the invariants of $X_{\alpha}$ in the space $\left\{x, y, y^{\prime}, \Lambda\right\}$, where $\Lambda$ is the Lagrangian. The appropriate prolongation in this case is

$$
\text { pr } X=\xi \frac{\partial}{\partial x}+\eta \frac{\partial}{\partial y}+\zeta^{1} \frac{\partial}{\partial y^{\prime}}-(D \xi) \Lambda \frac{\partial}{\partial \Lambda}, \quad \zeta^{1}=D(\eta)-y^{\prime} D(\xi)
$$

and we require that $L\left(x, y, y^{\prime}\right)$ should satisfy

$$
\left.\operatorname{pr} X(\Lambda-L)\right|_{\Lambda=L}=0
$$

Each basis element $X_{\alpha}$ provides us with an equation of the form

$$
\xi_{\alpha} \frac{\partial L}{\partial x}+\eta_{\alpha} \frac{\partial L}{\partial y}+\zeta_{\alpha}^{1} \frac{\partial L}{\partial y^{\prime}}-L D\left(\xi_{\alpha}\right)=0
$$

Solve the partial differential equations (3.10). This will give with the general form of an invariant Lagrangian. It may involve arbitrary functions of the invariants of $X$.

2. Request that the Euler equation (3.2) should coincide with the equation we started from. This will further restrict the invariant Lagrangian and determine whether one exists.

If this procedure does not yield a suitable Lagrangian, then step 1 can be weakened. We can request that the Lagrangian be invariant under some subgroup of the symmetry group of the given ODE, rather then the entire group. We then go through step 2, then verify whether the obtained Lagrangian is divergence invariant under the entire group, or at least a larger subgroup. In any case, each divergence symmetry of the Lagrangian will provide a first integral of the ODE.

For ODEs the Lagrangian formalism is not the only integration method. The existence of a one-parameter symmetry group provides a reduction to a first-order ODE directly. The existence of a two-parameter symmetry group makes it possible to integrate in quadratures. An invariant Lagrangian provides an alternative. Indeed, assume that we know two first integrals

$$
f_{1}\left(x, y, y^{\prime}\right)=A, \quad f_{2}\left(x, y, y^{\prime}\right)=B
$$

then we eliminate $y^{\prime}$ from these two equations and obtain the general solution

$$
y=F(x, A, B)
$$

of the corresponding ODE (3.3) by purely algebraic manipulations. It is this method of invariant Lagrangians that generalizes to difference equations and is particularly useful when direct methods fail. 


\section{Lagrangian formalism for second order difference equa- tions}

The variational formulation of discrete equations and a discrete analog of Noether's theorem are much more recent $[19,25,26,27]$. Here we briefly overview the results that we shall need below.

Let us consider a finite difference functional

$$
\mathbb{L}_{h}=\sum_{\Omega} \mathcal{L}\left(x, x_{+}, y, y_{+}\right) h_{+}
$$

defined on some one-dimensional lattice $\Omega$ with step $h_{+}$that generally can depend on the solution

$$
h_{+}=\varphi\left(x, y, x_{-}, y_{-}, y_{+}\right) .
$$

The functional (4.1) must be considered together with the lattice (4.2). On different lattices it can have different continuous limits and in this limit the lattice equation itself vanishes (turns into an identity like $0=0$ )

In the continuous case, a Lagrangian $L$ provides an equation (the Euler-Lagrange equation) that inherits all the symmetries of $L$. In the discrete case we wish the Lagrangian $\mathcal{L}$ to provide two equations: the entire difference system (1.1),(1.2). The threepoint difference system should inherit the symmetries of the two-point Lagrangian.

Let us again consider a Lie group of point transformations, generated by a Lie algebra of vector fields $X_{\alpha}$ of the form (1.3). The infinitesimal invariance condition of the functional (4.1) on the lattice (4.2) is given by two equations:

$$
\begin{aligned}
& \underset{\xi}{\xi} \frac{\partial \mathcal{L}}{\partial x}+\xi^{+} \frac{\partial \mathcal{L}}{\partial x_{+}}+\eta \frac{\partial \mathcal{L}}{\partial y}+\eta^{+} \frac{\partial \mathcal{L}}{\partial y_{+}}+\underset{+h}{\mathcal{L}} \underset{+h}{D}(\xi)=0, \\
& \underset{+h}{S}(\xi)-\xi=X(\varphi)
\end{aligned}
$$

where

$$
\xi^{+}=\xi\left(x_{+}, y_{+}\right), \quad \eta^{+}=\eta\left(x_{+}, y_{+}\right) .
$$

Now let us consider a vector field (1.3) with given coefficients $\xi(x, y)$ and $\eta(x, y)$. It has been shown $[19,25,27]$, that a stationary value of the difference functional (4.1) along the flow generated by this vector field is given by the quasi-extremal equations:

$$
\xi \frac{\delta \mathcal{L}}{\delta x}+\eta \frac{\delta \mathcal{L}}{\delta y}=0
$$

which are explicitly dependent on the coefficients of the generator . 
If we have a Lie algebra of vector fields of dimension 2 or more, then a stationary value of the difference functional (4.1) along the entire flow will be achieved on the intersection of all quasi-extremals. This intersection of quasi-extremals is given by the two equations:

$$
\begin{aligned}
& \frac{\delta \mathcal{L}}{\delta x}=h_{+} \frac{\partial \mathcal{L}}{\partial x}+h_{-} \frac{\partial \mathcal{L}^{-}}{\partial x}+\mathcal{L}^{-}-\mathcal{L}=0, \\
& \frac{\delta \mathcal{L}}{\delta y}=h_{+} \frac{\partial \mathcal{L}}{\partial y}+h_{-} \frac{\partial \mathcal{L}^{-}}{\partial y}=0
\end{aligned}
$$

where $\mathcal{L}^{-}=\underset{-h}{S}(\mathcal{L})$. These equations do not depend on coefficients of generators. In the continuous limit both of these equations reduce to the continuous Euler-Lagrange equation (3.2).

On the other hand, equations (4.6) and (4.7) can be interpreted as a three-point difference scheme of the form (1.1),(1.2). For instance, given two points $(x, y)$ and $\left(x_{-}, y_{-}\right)$, we can calculate $\left(x_{+}, y_{+}\right)$. In the continuous limit both of these equations will provide the same second-order differential equation.

It has been shown elsewhere $[19,25,27]$, that if the functional (4.1) is invariant under some group $G_{r}$, then the intersection (4.6) and (4.7) of the quasi-extremal equations is also invariant with respect to $G_{r}$. As in the continuous case, the intersection equations can be invariant with respect to a larger group than the corresponding Lagrangian.

A useful operator identity, valid for any Lagrangian $\mathcal{L}\left(x, x_{+}, y, y_{+}\right)$and any vector field $X$ is $([19,25])$ :

$$
\begin{aligned}
& \xi \frac{\partial \mathcal{L}}{\partial x}+\xi^{+} \frac{\partial \mathcal{L}}{\partial x_{+}}+\eta \frac{\partial \mathcal{L}}{\partial y}+\eta^{+} \frac{\partial \mathcal{L}}{\partial y_{+}}+\underset{+h}{\mathcal{L}}(\xi) \\
& =\xi\left(\frac{\partial \mathcal{L}}{\partial x}+\frac{h_{-}}{h_{+}} \frac{\partial \mathcal{L}^{-}}{\partial x}-\underset{+h}{D}\left(\mathcal{L}^{-}\right)\right)+\eta\left(\frac{\partial \mathcal{L}}{\partial y}+\frac{h_{-}}{h_{+}} \frac{\partial \mathcal{L}^{-}}{\partial y}\right) \\
& +\underset{+h}{D}\left(h_{-} \eta \frac{\partial \mathcal{L}^{-}}{\partial y}+h_{-} \xi \frac{\partial \mathcal{L}^{-}}{\partial x}+\xi \mathcal{L}^{-}\right) .
\end{aligned}
$$

From Eq. (4.8) we obtain the following discrete analog of Noether's theorem.

Theorem 1. ([19,25]) Let the Lagrangian $\mathcal{L}$ be invariant under a Lie group $G_{r}$ of dimension $r \geq 2$. Let the quasi-extremal equations (4.6), (4.7) be satisfied. Then each basis element $X_{\alpha}$ of the Lie algebra generating the group $G$ will provide us with a first integral for the intersection of the quasi-extremal equations (4.6) and (4.7), namely

$$
I_{\alpha}=h_{-} \eta_{\alpha} \frac{\partial \mathcal{L}^{-}}{\partial y}+h_{-} \xi_{\alpha} \frac{\partial \mathcal{L}^{-}}{\partial x}+\xi_{\alpha} \mathcal{L}^{-}, \quad 1 \leq \alpha \leq r .
$$


Similarly as in the continuous case, the invariance condition for the Lagrangian can be weakened and the result of Theorem 1 strengthened $[19,25]$. Indeed, let there exist a function $V=V(x, y)$ such that the discrete Lagrangian satisfies

$$
\operatorname{pr} X(\mathcal{L})+\underset{+h}{\mathcal{L}} \underset{+h}{D}(\xi)=\underset{+h}{D}(V)
$$

i.e. the Lagrangian is divergence invariant under $X$, rather than invariant. Then the symmetry $X$ generates a first integral for the system (4.6), (4.7) given by

$$
I=h_{-} \eta \frac{\partial \mathcal{L}^{-}}{\partial y}+h_{-} \xi \frac{\partial \mathcal{L}^{-}}{\partial x}+\xi \mathcal{L}^{-}-V .
$$

Eq. (4.11) is a discrete analog of Eq. (3.7) for ODEs. The condition (4.10) should hold on the solutions of the quasi-extremal equations (4.6) and (4.7).

Let us compare the situation for second order ODEs and for three point difference schemes. For a second order ODE a Lagrangian that is divergence invariant under a two-dimensional symmetry group provides two integrals of motion. From them we can eliminate the remaining first derivative and obtain the general solution, depending on two arbitrary constants (the two first integrals). Moreover, we do not really need a Lagrangian. Once we have a two dimensional symmetry algebra, we can integrate in quadratures.

For three-point difference schemes we have two equations to solve, namely the system $(1.1),(1.2)$. Equivalently, we have a set of points $\left(x_{n}, y_{n}\right)$, labeled by an integer $n$. Any 3 neighboring points are related by two equations that we can write e.g. as

$$
y_{n+1}=F_{1}\left(x_{n}, y_{n}, x_{n-1}, y_{n-1}\right), \quad x_{n+1}=F_{2}\left(x_{n}, y_{n}, x_{n-1}, y_{n-1}\right) .
$$

Alternatively, the system could be solved for $x_{n-1}, y_{n-1}$.

Given some starting values $\left(x_{0}, y_{0}, x_{1}, y_{1}\right)$, we can solve (4.12) for $\left(x_{n}, y_{n}\right)$ with $n \geq 2$, and $n \leq-1$. The solution will depend on four constants $K_{i}, i=1, \ldots, 4$ and can be written as

$$
\begin{aligned}
& y_{n}=y_{n}\left(x_{n}, K_{1}, K_{2}, K_{3}, K_{4}\right), \\
& x_{n}=x_{n}\left(K_{1}, K_{2}, K_{3}, K_{4}\right) .
\end{aligned}
$$

A one-parameter symmetry group of the Lagrangian $\mathcal{L}$ will provide us with one first integral, i.e. an equation of the form

$$
f\left(x_{n}, y_{n}, x_{n+1}, y_{n+1}\right)=I .
$$

compatible with the system (4.12). We can solve (4.15) for e.g. $y_{n+1}$, substitute into (4.12) and thus simplify this system.

A two-dimensional symmetry group will provide two first integrals of the form (4.15). We can solve for $x_{n+1}$ and $y_{n+1}$. Then system (4.12) is reduced to a two-point difference 
scheme. Quite often it is possible to obtain ad hoc integration methods that allow one to integrate a two-point difference scheme explicitly.

A three-dimensional symmetry group provides three equations of the type (4.15). From them we can express $x_{n-1}, y_{n-1}$ and $y_{n}$ in terms of $x_{n}$. This provides us with the solution (4.13) and a two-point difference equation relating $x_{n+1}$ and $x_{n}$. If this equation can be solved, we have a complete solution of the problem. Finally, if we have four first integrals, then we get the general solution of (4.12) by purely algebraic manipulations.

An alternative method can be proposed when the Lagrangian is invariant with respect to a two-dimensional Lie group. We can then require that the two quasi-extremal equations (4.6) and (4.7) be satisfied simultaneously. Moreover, the discrete Lagrangian corresponding to a given continuous one is not unique and it is possible to introduce a family of Lagrangians:

$$
\mathcal{L}_{i}=\mathcal{L}_{i}\left(x, x^{+}, y, y^{+}, \alpha_{i}, \beta_{i}\right), \quad i=1,2,3, \ldots
$$

depending on parameters $\alpha_{i}, \beta_{i}$, all satisfying

$$
\lim _{\left(x^{+}, y^{+}\right) \rightarrow(x, y)} \mathcal{L}_{i}\left(x, x^{+}, y, y^{+}, \alpha_{i}, \beta_{i}\right)=L\left(x, y, y^{\prime}\right)
$$

for the same continuous Lagrangian $L\left(x, y, y^{\prime}\right)$.

Let us take three different Lagrangians in the family (4.16), corresponding to constants $\alpha_{1}, \beta_{1}, \alpha_{2}, \beta_{2}$ and $\alpha_{3}, \beta_{3}$. Each of them leads to the two first integrals and two quasi-extremals. In examples considered below we will show that it is possible to fine-tune the constants $\alpha_{i}, \beta_{i}$ in such a manner as to get a system of three invariant equations (quasi-extremals) of the form (1.1),(1.2) and three first integrals, yielding a set of solutions to two of the quasi-extremal equations. It is these two equations that will constitute the invariant difference system.

We described a procedure for obtaining invariant Lagrangians for given second-order differential equations. For difference equations our starting point will be a discretization of the continuous Lagrangian. This is obviously not unique and we shall make use of the inherent arbitrariness. Once an invariant difference Lagrangian with correct continuous limit is chosen we construct the invariant difference scheme in the algorithmic manner described above.

\section{$5 \quad$ Example of the integration procedure}

In this section we present an example of a difference scheme with a three-dimensional symmetry algebra and a set of Lagrangians, all invariant under a two-dimensional subgroup of the symmetry group. Three different Lagrangians are used jointly to integrate the difference scheme analytically. 
The Lie algebra

$$
X_{1}=\frac{\partial}{\partial x}, \quad X_{2}=\frac{\partial}{\partial y}, \quad X_{3}=x \frac{\partial}{\partial x}+(x+y) \frac{\partial}{\partial y}
$$

corresponds to the invariant equation

$$
y^{\prime \prime}=\exp \left(-y^{\prime}\right) \text {. }
$$

The equation can be obtained from the Lagrangian

$$
L=\exp \left(y^{\prime}\right)+y
$$

which admits operators $X_{1}$ and $X_{2}$ :

$$
\begin{aligned}
& X_{1} L+L D\left(\xi_{1}\right)=0 ; \\
& X_{2} L+L D\left(\xi_{2}\right)=1=D(x) ;
\end{aligned}
$$

not however operator $X_{3}$.

It is possible to show that there is no Lagrangian which admits all three operators as divergence symmetries. Meanwhile, two different variational symmetries give two functionally independent first integrals that are sufficient to integrate a second order ODE.

Two first integrals given by the operators $X_{1}$ and $X_{2}$

$$
\exp \left(y^{\prime}\right)\left(1-y^{\prime}\right)+y=A^{0}, \quad \exp \left(y^{\prime}\right)-x=B^{0},
$$

where $A^{0}$ and $B^{0}$ are arbitrary constants, permit us to integrate the equation:

$$
y=\left(x+B^{0}\right)\left(\ln \left(x+B^{0}\right)-1\right)+A^{0} .
$$

\section{Invariant difference model and Lagrange-type integrability.}

Let us construct an integrable difference scheme, which can be represented by means of the following difference invariants for the operators (5.1):

$$
\frac{h_{+}}{h_{-}}, \quad y_{x}-y_{\bar{x}}, \quad \frac{1}{h_{-}} \exp \left(y_{\bar{x}}\right) .
$$

Let us choose three different difference Lagrangians in the following form:

$$
\mathcal{L}_{i}=\alpha_{i} \exp \left(y_{x}\right)+\gamma_{i} y+\left(1-\gamma_{i}\right) y_{+}, \quad i=1,2,3,
$$

where $\alpha_{i}$ and $\gamma_{i}$ are constants, which will be determined later. For these Lagrangians we have:

$$
\begin{aligned}
X_{1} \mathcal{L}_{i}+\underset{+h}{\mathcal{L}_{i} D}\left(\xi_{1}\right) & =0, \\
X_{2} \mathcal{L}_{i}+\underset{+h}{\mathcal{L}_{i} \underset{+h}{D}\left(\xi_{2}\right)}=1 & =\underset{+h}{D}(x),
\end{aligned}
$$


for any $\alpha_{i}, \gamma_{i}, \mathrm{i}=1,2,3$.

We will use different coefficients $\alpha_{i}, \gamma_{i}$ (this means different approximations of the continuous Lagrangian) for variations along different orbits of the group of translations along $x$ and $y$. The third invariant Lagrangian will be used to derive an invariant mesh. In fact we will use some small invariant deviation of the Lagrangian

$$
\mathcal{L}_{3}=\mathcal{L}_{1}+\delta_{1} \exp \left(y_{x}\right)+\delta_{2} y-\delta_{2} y_{+},
$$

where $\delta_{1}$ and $\delta_{2}$ tend to zero together with spacings $h^{+}$and $h^{-}$.

The variations of $\mathcal{L}_{i}$ yield in general six quasi-extremal equations. We choose three of them, namely:

$$
\begin{array}{ll}
\frac{\delta \mathcal{L}_{1}}{\delta y}: & \alpha_{1}\left(\exp \left(y_{x}\right)-\exp \left(y_{\bar{x}}\right)\right)=\gamma_{1} h^{+}+\left(1-\gamma_{1}\right) h^{-} \\
\frac{\delta \mathcal{L}_{2}}{\delta x}: & \alpha_{2} \exp \left(y_{x}\right)\left(y_{x}-1\right)-\alpha_{2} \exp \left(y_{\bar{x}}\right)\left(y_{\bar{x}}-1\right) \\
=\gamma_{2} y+\left(1-\gamma_{2}\right) y_{+}-\gamma_{2} y_{-}-\left(1-\gamma_{2}\right) y
\end{array}
$$

The two first equations approximate the corresponding continuous Euler's equation, and the third equation generates a mesh:

$$
\frac{\delta \mathcal{L}_{3}}{\delta y}: \alpha_{3}\left(\exp \left(y_{x}\right)-\exp \left(y_{\bar{x}}\right)\right)=\gamma_{3} h^{+}+\left(1-\gamma_{3}\right) h^{-} .
$$

Due to the invariance of the Lagrangian with respect to the operators $X_{1}$ and $X_{2}$, the difference analog of Noether's identity yields the following first integrals

$$
\begin{aligned}
& \alpha_{1} \exp \left(y_{x}\right)=x+\gamma_{1} h^{+}+B^{h}, \\
& \alpha_{2} \exp \left(y_{x}\right)\left(y_{x}-1\right)=y+\left(1-\gamma_{2}\right) h^{+} y_{x}+A^{h}, \\
& \left(\alpha_{1}-\alpha_{3}\right) \exp \left(y_{x}\right)=\left(\gamma_{1}-\gamma_{3}\right) h^{+}+\left(B^{h}-B_{h}\right),
\end{aligned}
$$

where we wrote the third integral for the difference between the first and third quasiextremal equations.

Now our task is to develop the intersection of the quasi-extremals (5.5),(5.6), or, equivalently, the intersection of the first integrals $(5.7),(5.8)$ (i.e. to search for the needed constants). The compatibility of (5.7),(5.8) yields the value of the constants $\alpha_{i}, \gamma_{i}$, which can be the following:

$$
\begin{aligned}
\alpha_{1} & =1, \quad \alpha_{2}=\ln (1+\varepsilon) \varepsilon^{-1}, \\
\gamma_{1} & =1, \quad \gamma_{2}=1-(1+\varepsilon) \varepsilon^{-2} \ln (1+\varepsilon)+\varepsilon^{-1} . \\
B^{h} & =B_{h}, \quad \frac{\alpha_{1}-\alpha_{3}}{\gamma_{1}-\gamma_{3}}=\frac{\varepsilon}{1+\varepsilon} .
\end{aligned}
$$


Thus, the mesh equation is:

$$
h^{+}=\frac{\varepsilon \exp \left(y_{x}\right)}{1+\varepsilon} .
$$

With the obtained constants the equations (5.5),(5.6) can be integrated by means of first integrals. The general solution is:

$$
y=\left(x+B^{h}\right) \ln \left(x+B^{h}\right)-\left(1+\varepsilon^{2}\right)\left(x+B^{h}\right)+A^{h} .
$$

This solution is considered on the mesh (5.11), which is equivalent (on the solution (5.12)) to

$$
h_{+}=\varepsilon\left(x+B^{h}\right) .
$$

The last one is linear and can easily be integrated for the whole set of mesh points:

$$
x_{n}=(1+\varepsilon)^{n}\left(x_{0}+B^{h}\right)-B^{h},
$$

where $n=0,1,2, \ldots$, and $x_{0}$ is any starting point.

So, as an invariant difference approximation of (5.2) we can consider the following:

$$
\left\{\begin{array}{c}
\frac{1}{h_{+}}\left(\exp \left(y_{x}\right)-\exp \left(y_{\bar{x}}\right)\right)=1 \\
h_{+} \exp \left(-y_{x}\right)=h_{-} \exp \left(-y_{\bar{x}}\right) .
\end{array}\right.
$$

Notice, that the last equation on the solution (5.12) again can be rewritten as some exponential mesh (q-mesh):

$$
h_{+}=(1+\varepsilon) h^{-} .
$$

This scheme is invariant with respect to all symmetries (5.1) and possesses the integrals:

$$
\begin{aligned}
& \exp \left(y_{x}\right)=x+h^{+}+B^{h}, \\
& \frac{\ln (1+\varepsilon)}{\varepsilon} \exp \left(y_{x}\right)\left(y_{x}-1\right)=y+\left((1+\varepsilon) \varepsilon^{-2} \ln (1+\varepsilon)-\varepsilon^{-1}\right) h^{+} y_{x}+A^{h}, \\
& \frac{\varepsilon}{(1+\varepsilon)} \exp \left(y_{x}\right)=h^{+} .
\end{aligned}
$$

The solution (5.12),(5.14) depends on the four constants $\varepsilon, A^{h}, B^{h}, x_{0}$. All these constants can be obtained from initial conditions $\left(x_{0}, y_{0}, x_{1}, y_{1}\right)$ for the system (5.15).

Notice that the invariant mesh was developed without additional symmetry of the Lagrange function, but by means of a small invariant perturbation of an invariant Lagrangian. 


\section{Concluding remarks}

We constructed variational discrete models which approximate the equations from Lie's classification of second order ODEs. The variational formulation makes it possible to use Noether's theorem. With the help of symmetries one can find first integrals which can be used to reduce the order of the discrete model. We observed the following possibilities for order reduction corresponding to the different number of variational symmetries:

1. For one symmetry we can get only one first integral with the help of the Lagrangian approach. It holds on the extremal equation (4.5) considered on some invariant mesh. In this case there is no need to consider the intersection of the quasi-extremal equations.

2. Two symmetries provide us with two functionally independent first integrals $I_{1}$, $I_{2}$, which hold on the intersection of the quasi-extremals. These first integrals reduce the system to the form

$$
\begin{aligned}
& \tilde{F}\left(x, x_{+}, y, y_{+}, I_{1}, I_{2}\right)=0, \\
& \tilde{\Omega}\left(x, x_{+}, y, y_{+}, I_{1}, I_{2}\right)=0 .
\end{aligned}
$$

3. Three symmetries let us find three independent first integrals $I_{1}, I_{2}, I_{3}$. It is possible to reduce the system of the quasi-extremal equation to a mapping of the from

$$
x_{+}=f\left(x, I_{1}, I_{2}, I_{3}\right) \quad \text { or } \quad y_{+}=g\left(y, I_{1}, I_{2}, I_{3}\right)
$$

with an equation $H\left(x, y, I_{1}, I_{2}, I_{3}\right)=0$ connecting $x$ and $y$. If the mapping is integrable, we can write out the general solution of the discrete model (4.6), (4.7) in the form

$$
x_{i}=X\left(i, x_{0}, y_{0}, x_{1}, y_{1}\right), \quad y_{i}=Y\left(i, x_{0}, y_{0}, x_{1}, y_{1}\right)
$$

satisfying the initial conditions

$$
\begin{aligned}
& X\left(0, x_{0}, y_{0}, x_{1}, y_{1}\right)=x_{0}, \quad Y\left(0, x_{0}, y_{0}, x_{1}, y_{1}\right)=y_{0} \\
& X\left(1, x_{0}, y_{0}, x_{1}, y_{1}\right)=x_{1}, \quad Y\left(1, x_{0}, y_{0}, x_{1}, y_{1}\right)=y_{1}
\end{aligned}
$$

The approach can be extended in many directions. It is possible to extend the presented framework to the case of several dependent variables and higher order ODEs. Discrete models which do not have a continuous limit, but can be presented as quasiextremal equations for an appropriate discrete Lagrangian, are also covered by the discrete Lagrangian formalism. A complete study of the Lagrangian formalism for second order difference schemes will be published elsewhere [32]. 


\section{Acknowledgments}

The research of V.D. was partially sponsored by Russian Fund for Basic Research, the research of R.K. by The Norwegian Research Council under contracts no.111038/410, through the SYNODE project, and no.135420/431, through the BeMatA program, and the research of P.W. by research grants from NSERC of Canada and FCAR du Quebec. V.D. and P.W. benefited from a NATO Collaborative Linkage Grant that made our collaboration possible.

\section{References}

[1] V.Dorodnitsyn, R.Kozlov, P.Winternitz, Lie group classification of second order difference equations, J. Math. Phys. 41(1) (2000), 480-504

[2] S.Lie, Klassifikation und Integration von Gewohnlichen Differentialgleichunden zwischen $x, y$ die eine Gruppe von Transformationen gestatten, Math. Ann. 32 (1888), 213-281, Leipzig, 1924.

[3] S.Maeda, Canonical structure and symmetries for discrete systems, Math. Japan $\mathbf{2 5}$ (1980), 405

[4] D.Levi and P.Winternitz, Continuous symmetries of discrete equations, Phys. Lett. A152 (1991), 335

[5] D.Levi and P.Winternitz, Symmetries and conditional symmetries of differential-difference equations, J. Math. Phys. 34 (1993), 3713

[6] D.Levi and P.Winternitz, Symmetries of discrete dynamical systems, J. Math. Phys. 37 (1996), 5551

[7] D.Levi, L.Vinet, and P.Winternitz, Lie group formalism for difference equations, J. Phys. A Math. Gen. 30 (1997), 663

[8] R.Hernandez-Heredero, D.Levi and P.Winternitz, Symmetries of the discrete Burgers equation, J. Phys. A Math. Gen. 32 (1999), 2685

[9] D.Gomez-Ullate, S.Lafortune, and P.Winternitz, Symmetries of discrete dynamical systems involving two species, J. Math. Phys. 40 (1999), 2782

[10] R.Hernandez-Heredero, D.Levi, M.A.Rodriguez and P.Winternitz, Lie algebra contractions and symmetries of the Toda hierarchy, J. Phys. A Math. Gen. 33 (2000), 5025

[11] D.Levi, S.Tremblay and P.Winternitz, Lie point symmetries of difference equations and lattices, J. Phys. A Math. Gen. 33 (2000), 8507

[12] D.Levi, S.Tremblay and P.Winternitz, Lie symmetries of multidimensional difference equations, J. Phys. A Math. Gen. 34 (2001), 9507

[13] D.Levi and P.Winternitz, Lie point symmetries and commuting flows for equations and lattices, J. Phys. A Math. Gen. 35 (2002), 2249 
[14] R.Floreanini, J.Negro, L.M.Nieto and L.Vinet, Symmetries of the heat equation on a lattice, Lett. Math. Phys. 36 (1996), 351

[15] R.Floreanini and L.Vinet, Lie symmetries of finite-difference equations, J. Math. Phys. 36 (1995), 7024

[16] G.R.W.Quispel, H.W.Capel, and R. Sahadevan, Continuous symmetries of difference equations; the Kac - van Moerbeke equation and Painleve reduction, Phys. Lett. A170 (1992), 379

[17] V.A.Dorodnitsyn, Transformation groups in a space of difference variables, in VINITI Acad. Sci. USSR, Itogi Nauki i Techniki, 34 (1989), 149-190, (in Russian), see English transl. in J. Sov. Math. 55 (1991), 1490

[18] V.Dorodnitsyn, Finite-difference models entirely inheriting the symmetry of the original differential equations Modern Group Analysis: Advanced Analytical and Computational Methods in Mathematical Physics, Kluwer Academic Publishers (1993), 191

[19] V.A.Dorodnitsyn, Finite-difference analog of the Noether theorem, Dokl. Akad. Nauk, 328 (1993), 678 (in Russian).

[20] V.A.Dorodnitsyn, Finite-difference models entirely inheriting continuous symmetry of original differential equations, Int. J. Mod. Phys. C, (Phys. Comp.), 5 (1994), 723

[21] V.Dorodnitsyn, Continuous symmetries of finite-difference evolution equations and grids, in Symmetries and Integrability of Difference Equations, CRM Proceedings and Lecture Notes, Vol. 9, AMS, Providemce, R.I., 103-112 (1996), Ed. by D.Levi, L.Vinet and P.Winternitz, see also V.Dorodnitsyn, Invariant discrete model for the Korteweg-de Vries equation, Preprint CRM-2187, Montreal (1994)

[22] W.F.Ames, R.L.Anderson, V.A.Dorodnitsyn, E.V.Ferapontov, R.K.Gazizov, N.H.Ibragimov and S.R.Svirshchevskii, CRC Hand-book of Lie Group Analysis of Differential Equations, ed. by N.Ibragimov, Volume I: Symmetries, Exact Solutions and Conservation Laws, CRC Press, 1994.

[23] M.Bakirova, V.Dorodnitsyn and R.Kozlov, Invariant difference schemes for heat transfer equations with a source, J. Phys. A: Math. Gen., 30 (1997), 8139 see also

V.Dorodnitsyn and R.Kozlov, The complete set of symmetry preserving discrete versions of a heat transfer equation with a source, Preprint of NTNU, NUMERICS NO. 4/1997, Trondheim, Norway (1997)

[24] V.Dorodnitsyn and P.Winternitz, Lie point symmetry preserving discretizations for variable coefficient Korteweg-de Vries equations, CRM-2607, Nonlinear Dynamics, 22(1), Kluwer (2000), 49

[25] V.Dorodnitsyn, Noether-type theorems for difference equations, Applied Numerical Mathematics, 39 (2001), 307-321

[26] C.Budd, V.Dorodnitsyn, Symmetry-adapted moving mesh schemes for the nonlinear Schroedinger equation, J. Phys. A: Math. Gen., 34(48) (2001), 10387-10400 
[27] V.Dorodnitsyn, The Group Properties of Difference Equations, Moscow, Fizmatlit, 2001 (in Russian).

[28] E.Noether, Invariante Variationsprobleme, Nachr. Konig. Gesell. Wissen., Gottingen, Math.-Phys. Kl., Heft 2 (1918), 235

[29] E.Bessel-Hagen, Uber die Erhaltungssatze der Elektrodynamik. Math. Ann., 84 (1921), 258

[30] P.J.Olver, Applications of Lie Groups to Differential Equations, Springer, New York, 1986

[31] N.H.Ibragimov, Transformation Groups Applied to Mathematical Physics, Reidel, Boston, 1985

[32] V.Dorodnitsyn, R.Kozlov and P.Winternitz, Continuous symmetries of Lagrangians and exact solutions of discrete equations, J. Math. Phys., to appear. 